\title{
Association of Cognitive Function Screening Results with Adherence and Performance in a Pedometer-Based Intervention
}

\author{
Anoop Sheshadria,b Piyawan Kittiskulnam ${ }^{c, d}$ Cynthia Delgado ${ }^{a, b}$ \\ Rebecca L. Sudore ${ }^{e}$ Jennifer C. Laif Kirsten L. Johanseng, h \\ ${ }^{a}$ Nephrology Section, San Francisco Veterans Affairs Medical Center, San Francisco, CA, USA; ${ }^{b}$ Division of \\ Nephrology, Department of Medicine, University of California, San Francisco, CA, USA; 'Division of Internal \\ Medicine-Nephrology, Department of Medicine, Chulalongkorn University, Bangkok, Thailand; ${ }^{\mathrm{d}}$ Special Task \\ Force for Activating Research in Renal Nutrition (Renal Nutrition Research Group), Office of Research Affairs, \\ Chulalongkorn University, Bangkok, Thailand; 'Division of Geriatrics, Department of Medicine, San Francisco \\ Veterans Affairs Medical Center, University of California, San Francisco, CA, USA; fDivision of Gastroenterology/ \\ Hepatology, Department of Medicine, University of California, San Francisco, CA, USA; 9Division of Nephrology, \\ Hennepin County Medical Center, Minneapolis, MN, USA; hivision of Nephrology, University of Minnesota, \\ Minneapolis, MN, USA
}

\section{Keywords}

Physical activity $\cdot$ Exercise $\cdot$ Dialysis $\cdot$ Motivations $\cdot$ Barriers

\begin{abstract}
Introduction: A randomized, controlled trial of a pedometer-based walking intervention with weekly activity goals led to increased walking among dialysis patients. We examined whether impairment per cognitive function screening is associated with adherence and performance in the intervention. Methods: Thirty dialysis patients were randomly assigned to a 3-month pedometer-based intervention with weekly goals. Participants were administered the Telephone Interview of Cognitive Status (TICS), a test of global mental status. We examined the association of levels of impairment on the TICS ( $\geq 33$ : unimpaired, 26-32: ambiguous impairment, 21-25: mild cognitive impairment [MCl]) with adherence, achieving weekly goals, and increasing steps, physical
\end{abstract}

karger@karger.com www.karger.com/ajn

Karger $\stackrel{\text { ' }}{5}$

GOPEN ACCESS
(C) 2021 The Author(s)

Published by S. Karger AG, Basel

This is an Open Access article licensed under the Creative Commons Attribution-NonCommercial-4.0 International License (CC BY-NC) (http://www.karger.com/Services/OpenAccessLicense), applicable to the online version of the article only. Usage and distribution for commercial purposes requires written permission. performance (Short Physical Performance Battery, SPPB), and self-reported physical function (PF) through multivariable linear mixed-model and logistic regression analyses adjusted for age, sex, BMl, dialysis modality, baseline steps, baseline SPPB, and stroke status. Results: One-third of participants were unimpaired, and $13 \%$ had $\mathrm{MCl}$. Participants with worse results on cognitive function screening missed more calls and completed fewer weekly goals than participants with better results. During the intervention, a worse result on cognitive function screening was associated with smaller increases in steps compared to those without impairment: (ambiguous: -620 [95\% Cl -174, -1,415], MCl: $-1,653$ $[95 \% \mathrm{Cl}-120,-3,187]$ ); less improvement in SPPB (ambiguous: -0.22 points $[95 \% \mathrm{Cl}-0.08,-0.44], \mathrm{MCl}:-0.45[95 \% \mathrm{Cl}$ $-0.13,-0.77]$ ); and less improvement in PF (ambiguous: -4.0 points [95\% Cl $-12.2,4.1], \mathrm{MCl}:-14.0[95 \% \mathrm{Cl}-24.9,-3.1]$ ). During the postintervention period, a worse result on cognitive function screening was associated with smaller increas- 
es in SPPB (ambiguous: -0.54 [95\% Cl-1.27, 0.19], MCl: -0.97 [95\% Cl-0.37, -1.58$]$ ) and PF (ambiguous: -3.3 [95\% Cl-6.5, $-0.04], \mathrm{MCl}:-10.5$ [95\% Cl-18.7, -2.3]). Discussion/Conclu-

sion: Participants with worse results on cognitive function screening had worse adherence and derived less benefit from this pedometer-based intervention. Future exercise interventions should be developed incorporating methods to address cognitive impairment, for example, by including caregivers when planning such interventions.

(C) 2021 The Author(s)

Published by S. Karger AG, Basel

\section{Introduction}

End-stage kidney disease (ESKD) can be considered a disease of accelerated or accentuated aging, and patients often have deficits in cognitive and physical functioning $[1,2]$. The pathophysiology of cognitive impairment in ESKD is likely multifactorial, including cerebrovascular disease - particularly common in patients treated with dialysis [3-5] - as well as metabolic causes such as oxidative stress, anemia, and malnutrition [6-8]. Patients with ESKD have nearly double the prevalence of cognitive dysfunction as the general population, even after accounting for age, diabetes, cardiovascular risk, and other comorbid conditions [9-11]. Furthermore, even mild to moderate cognitive impairment is associated with higher mortality among patients with ESKD compared to those without impairment [6, 12-14]. In the general elderly population, lower levels of physical activity are associated with impaired cognitive function $[15,16]$. However, many patients treated with dialysis report extremely sedentary levels of activity irrespective of age, well below those in the general elderly population [17-20]. Tailoring interventions to accommodate impairments in cognitive function among patients with ESKD may be key to improving physical function and activity, but this possibility has not been examined.

Indeed, physical activity and exercise interventions are feasible and effective in improving physical function in older individuals in the general population despite baseline mild cognitive impairment or even dementia [21]. Evidence derived from the non-ESKD population suggests that participants with cognitive impairment may have improved "trainability" and adherence to physical activity interventions if specific measures are taken to mitigate the effect of cognitive impairment [2225]. Although there is also a significant body of evidence in support of exercise interventions to improve physical activity and function in patients with ESKD, the major- ity of these do not take cognitive impairment into account [26-28]. Furthermore, although there are general guidelines for activity interventions for the cognitively impaired [29], there are little data on the efficacy of protocols for patients with overlapping physical and cognitive impairments such as those with ESKD [30]. Such information may be especially valuable when designing interventions for patients treated with dialysis, who are likely to have unique barriers to exercise and activity interventions that may amplify the effect of cognitive impairment $[31,32]$.

We conducted a study of a 6-month randomized controlled trial comparing 3 months of pedometers and weekly step goals plus a 3-month postintervention follow-up period without counseling or pedometers with usual care among patients treated with dialysis [20]. Although the study led to increased walking among participants assigned to the intervention, adherence and benefit were variable, and it was unclear whether this may be related to cognitive impairment. We conducted a secondary analysis among those in the intervention group to examine whether lower scores on cognitive function screening were associated with adherence to the intervention or with a difference in outcomes.

\section{Materials and Methods}

Inclusion and Exclusion Criteria

Patients were enrolled from 3 dialysis clinics in San Francisco. Individuals were included if they were 18 years of age or older, receiving dialysis treatment with either peritoneal dialysis (PD) or in-center hemodialysis (HD), having telephone access, and were ambulatory without the need for use of a wheelchair or scooter. With permission of the treating physician, patients were initially identified as potential candidates by dialysis staff, and then screened by study personnel for eligibility. All patients provided informed consent to participate and were required to summarize the intent of the study and the information from the consent form to study personnel prior to enrollment. The study was approved by the UCSF Committee on Human Research (14-13175) and was registered at ClinicalTrials.gov (NCT02623348).

\section{Baseline Testing and Step Counts}

Participants were asked their race and ethnicity, and medical records were reviewed for information about dialysis prescription, laboratory results, comorbid conditions, and medications. We measured step counts using pedometers (Accusplit AE120, Livermore, CA, USA), which is highly accurate at tracking steps through accelerometers detecting vertical movement at the hip [17, 33-36]. Patients were asked to wear the pedometer at their waist continuously during waking hours for 1 week and to record their daily steps. Step counts were relayed to study personnel in person or by telephone. 


\section{Randomization and Intervention}

Patients were randomly assigned to participate in a 3-month intervention or control group in a 1:1 ratio, stratified by dialysis modality. Full details of recruitment and enrollment have been previously described [20]. The intervention consisted of providing pedometers in conjunction with weekly counseling sessions in which a member of the study team called the participant at a scheduled time each week. Participants in the intervention were asked to wear their pedometers and to record their step counts in a daily log for the full 3 months. All participants were trained on how to use their pedometers prior to obtaining baseline steps. During the counseling sessions, participants reported their steps, and research personnel provided participants with specific goals for daily walking for the upcoming week and advised about ways to increase the amount of walking in the context of their daily routines.

Participants were recommended to increase their steps by $10 \%$ each week compared with the prior week. At the start of the intervention, each participant was given a graphical projection of the expected trajectory of their average daily step counts over the 12week intervention assuming successful increase of steps by $10 \%$ each week. This projected step count at 12 weeks was considered their overall target, to a maximum of 10,000 steps per day. In any given week, patients who did not meet their weekly target were not set a higher target for the subsequent week. We revised goals for any patients who had periods of reduced walking (e.g., after hospitalizations or other events) such that they would increase in $10 \%$ increments of their new "baseline" daily steps. Participants were given feedback about their revised projections each week but were not formally given a new overall target.

\section{Cognitive Function Screening}

All outcomes were measured at baseline, at 3 months (postintervention), and again at 6 months ( 3 months after completion of intervention). Our predictor, cognitive impairment, was measured through in-person administration of the Telephone Interview for Cognitive Status (TICS) [37], a 41-point global test for mental status with excellent correlations with more commonly used tests (such as the Mini-Mental Status Exam [MMSE]) [38] but without the MMSE's reliance on intact vision and upper extremity function [39]. Cognitive domains measured by the TICS include: orientation, concentration, short-term memory, language, praxis, and mathematical skills [38]. TICS scores can be used to categorize individuals into categories as follows: unimpaired [33-41], ambiguous impairment (i.e., further testing needed to determine whether cognitive impairment is present, 26-32), mild cognitive impairment [21-25], and moderate to severe cognitive impairment $(<21)[40]$.

\section{Physical Outcomes}

Physical performance was measured by the Short Physical Performance Battery (SPPB), an objective assessment of lower extremity function that includes gait speed, sit-to-stand testing, and balance testing [41]. We also measured self-reported physical function with the Physical Functioning (PF) Scale of the SF-36 [42]. Physical performance was assessed prior to HD for patients treated with $\mathrm{HD}$, and questionnaires were administered during the dialysis session. Patients on PD were assessed at a usual clinic visit. All interviews were conducted face to face, and questionnaires were administered according to established guidelines [40]. For
Table 1. Patient characteristics at baseline for 30 participants

\begin{tabular}{|c|c|}
\hline Characteristic & $\begin{array}{l}\text { Median } \\
\text { (25th, 75th percentile) }\end{array}$ \\
\hline Age, years & $60(53,66)$ \\
\hline Sex, $\%$ male & 93 \\
\hline Hispanic, \% & 17 \\
\hline \multicolumn{2}{|l|}{ Race, $\%$} \\
\hline White & 13 \\
\hline Black & 47 \\
\hline Asian & 20 \\
\hline Other & 20 \\
\hline BMI, $\mathrm{kg} / \mathrm{m}^{2}$ & $26.9(25.3,32.9)$ \\
\hline \multicolumn{2}{|l|}{ Comorbidities, $\%$} \\
\hline HTN & 93 \\
\hline $\mathrm{DM}$ & 33 \\
\hline CAD & 37 \\
\hline $\mathrm{CHF}$ & 30 \\
\hline Stroke & 7 \\
\hline Peripheral vascular disease & 13 \\
\hline Arrhythmia & 20 \\
\hline \multicolumn{2}{|l|}{ Dialysis modality, \% } \\
\hline Hemodialysis & 80 \\
\hline Peritoneal dialysis & 20 \\
\hline Dialysis vintage, years & $3.7(1.5,7.2)$ \\
\hline Hemoglobin, g/dL & $10.6(9.6,11.7)$ \\
\hline Serum albumin, g/dL & $3.9(3.6,4.1)$ \\
\hline Std Kt/V & $2.30(2.07,2.44)$ \\
\hline \multicolumn{2}{|l|}{ Education, \% } \\
\hline High school or less & 37 \\
\hline Vocational or some college & 33 \\
\hline College degree & 13 \\
\hline Professional or graduate degree & 20 \\
\hline Currently smoking, \% & 10 \\
\hline Use of assistive device, \% & 30 \\
\hline Cane & 20 \\
\hline Walker & 10 \\
\hline
\end{tabular}

step counts, we calculated average daily steps over the week prior to each assessment for each participant and reported the mean of those average daily steps. These instruments have been previously validated.

\section{Statistical Analysis}

Patients' baseline characteristics were summarized as median (25th, 75th percentile) for continuous variables and as frequency and percentage for categorical variables. Baseline steps were reported as mean (standard deviation). We used linear regression to assess the association between level of cognitive impairment and baseline steps, physical performance, and self-reported physical function. We also used linear regression to assess the association between level of cognitive impairment and adherence in the intervention including calls completed and goals met. We performed logistic regression to measure the association between cognitive impairment and odds of reaching the overall target set at the beginning of the intervention. 
Table 2. Association of cognitive function screening* with baseline step count, physical performance, and selfreported physical function

\begin{tabular}{|c|c|c|c|c|}
\hline Outcomes & Ambiguous impairment & $p$ value** & MCI & $p$ value $* *$ \\
\hline Average daily step count ${ }^{* * *}$ & $1,929(-5,206,1,348)$ & 0.24 & $-2,057(-6,599,2,485)$ & 0.36 \\
\hline $\mathrm{SPPB}^{\dagger}$ & $-1.74(-3.43,-0.05)$ & 0.04 & $-1.98(-4.32,0.35)$ & 0.09 \\
\hline $\mathrm{PF}^{\dagger}$ & $-21.7(-57.1,13.7)$ & 0.22 & $-41.7(-87.4,4.0)$ & 0.07 \\
\hline
\end{tabular}

${ }^{\dagger}$ SPPB, short physical performance battery; PF, physical functioning scale of the SF-36; MCI, mild cognitive impairment; TICS, telephone interview for cognitive status. ${ }^{*}$ Cognitive function defined per category of TICS (33-41 [unimpaired, reference], 26-32 [ambiguous impairment], and 21-25 [MCI]). ** $p$ value for linear regression, adjusted for age, sex, dialysis modality, BMI, stroke history, baseline step count, and baseline physical performance. ${ }^{* * *}$ Average daily step count was calculated by averaging individual daily step counts over the week prior to each assessment.

Finally, we used mixed effects linear regression analyses to estimate the association between level of cognitive impairment and change in step count, SPPB score, and PF from baseline to 3 months and from 3 to 6 months, and then performed analyses for linear trends across levels of cognitive impairment. All analyses were adjusted for age, sex, dialysis modality, BMI, and stroke history based on the prior literature. Because our prior study showed that baseline physical performance and baseline steps were associated with meeting weekly goals and targets [20], we additionally controlled for these factors.

Two-sided $p$ values $<0.05$ were considered statistically significant. Statistical analyses were performed using Stata, version 14 (StataCorp, College Station, TX, USA).

\section{Results}

\section{Baseline Characteristics and Step Counts during}

Intervention and Maintenance

There were $24 \mathrm{HD}$ and $6 \mathrm{PD}$ patients in the intervention group, aged 60 years $(53,66)$ at baseline, and the cohort was predominantly male (93\%) (Table 1). The cohort was diverse in terms of race, with $47 \%$ Black, $20 \%$ Asian, and 13\% White. Median BMI was 26.9 (25.3, 32.9). Hypertension was the most common comorbidity (93\%). Thirty percent of participants used an assistive device for walking. The median score on the TICS was 31 of 41 (25th and 75th percentile 28, 33). Thirtythree percent of those in the intervention had no cognitive impairment, $53 \%$ had ambiguous impairment, and $13 \%$ had MCI. No patient scored below 21 on the TICS (moderate to severe impairment). The average daily step count at baseline was 3,924 (SD 3,422), increased to $5,863(\mathrm{SD} 4,019)$ at the end of the 3-month intervention period, and fell back to 4,141 (SD 3,747) at 6 months. There was no significant change in cognitive function scores in either control or intervention groups over the course of the intervention ( 3 months) or at 6 months.

Association of Cognitive Function Scores with Physical Performance, Self-Reported Physical Function, and Baseline Steps

In multivariable analysis, participants with ambiguous impairment had worse physical performance at baseline than those without cognitive impairment (difference in SPPB - 1.74 [95\% CI -3.42, -0.05]; $p=0.04$ ) (Table 2). However, the association between MCI and physical performance was not statistically significant (difference in SPPB $-1.9895 \%$ CI $[-4.32,0.35] ; p=0.09$ ), and there was no statistically significant association between any level of cognitive impairment and baseline self-reported physical function or baseline step count.

\section{Association of Cognitive Function Scores with \\ Adherence}

Adherence was high, with $90 \%$ of participants completing the 3-month intervention. Eighty-three percent of all calls were completed as planned. Participants achieved a median of $33 \%(25,67)$ of their weekly step goals (i.e., met their weekly target during 4 of the 12 weeks of the intervention), and $37 \%$ of participants were able to achieve their overall target at the end of 12 weeks.

In multivariable analysis, participants who had a higher degree of cognitive impairment were more likely to miss calls (ambiguous impairment: $-20.2 \%$ successful calls [95\% CI $-40.1 \%,-0.05 \%] ; p=0.05$ and MCI: $-24.7 \%$ successful calls $[95 \% \mathrm{CI}-38.0 \%,-11.5 \%])$ than participants with better cognitive function. Participants with a 
Table 3. Association of cognitive function screening* with change in step count, physical performance, grip strength, and self-reported physical function from baseline to 3 months and from 3 to 6 months

\begin{tabular}{|c|c|c|c|c|c|c|}
\hline \multirow{2}{*}{ Outcomes: } & \multicolumn{3}{|l|}{ Baseline to 3 months } & \multicolumn{3}{|l|}{ Baseline to 3-6 months } \\
\hline & $\begin{array}{l}\text { association of cognitive fu } \\
\text { outcome }^{*}(95 \% \mathrm{CI})\end{array}$ & nction with change in & $\begin{array}{l}p \\
\text { value** }\end{array}$ & $\begin{array}{l}\text { association of cognitive } \mathrm{f} \\
\text { outcome* }(95 \% \mathrm{CI})\end{array}$ & unction with change in & $\begin{array}{l}p \\
\text { value** }\end{array}$ \\
\hline TICS category & Ambiguous impairment & MCI & & Ambiguous impairment & MCI & \\
\hline Average daily step count ${ }^{* * *}$ & $-620(-174,-1,415)$ & $-1,653(-120,-3,187)$ & $<0.01$ & $-521(-2,877,1,835)$ & $-738(-1,527,51)$ & 0.7 \\
\hline $\mathrm{SPPB}^{\dagger}$ & $-0.22(-0.08,-0.44)$ & $-0.45(-0.13,-0.77)$ & $<0.01$ & $-0.54(-1.27,0.19)$ & $-0.97(-0.37,-1.58)$ & 0.02 \\
\hline $\mathrm{PF}^{\dagger}$ & $-4.0(-12.2,4.1)$ & $-14.0(-24.9,-3.1)$ & 0.01 & $-3.3(-6.5,-0.04)$ & $-10.5(-18.7,-2.3)$ & 0.04 \\
\hline
\end{tabular}

${ }^{\dagger}$ SPPB, short physical performance battery; PF, physical functioning scale of the SF-36; MCI, mild cognitive impairment; TICS, telephone interview for cognitive status. ${ }^{*}$ Cognitive function defined per category of TICS (33-41 [unimpaired, reference], 26-32 [ambiguous impairment], <26 [impaired]), adjusted for age, sex, dialysis modality, BMI, stroke history, baseline step count, and baseline physical performance. ${ }^{* *} p$ value for linear test of trend across categories of cognitive impairment. ${ }^{* * *}$ Average daily step count was calculated by averaging individual daily step counts over the week prior to each assessment.

higher degree of cognitive impairment also completed fewer of their individual weekly goals (ambiguous impairment: $-23.8 \%$ goal completion [95\% CI $-41.8 \%$, $-0.06 \%] ; p=0.01$ and MCI: $-40.1 \%$ goal completion [95\% CI $-65.4 \%,-14.9 \%] ; p<0.01)$ and were less likely to achieve their overall targets (ambiguous impairment: OR 0.21 [95\% CI $0.05,0.99$ ]; $p=0.05$ ) than participants with better cognitive function. No participant with MCI met their overall target.

\section{Association of Cognitive Function Scores with Change} in Outcomes from Baseline to 3 months

Lower cognitive function on the TICS was associated with a smaller increase in physical performance on the SPPB (ambiguous impairment: -0.22 [95\% CI -0.08 , -0.44 ]; MCI: -0.45 [95\% CI $-0.13,-0.77$ ]; $p$ for trend $<0.01$ ), a smaller increase in self-reported physical function (ambiguous impairment: -4.0 [95\% CI -12.2, 4.1]; MCI: -14.0 [95\% CI $-24.9,-3.1] ; p$ for trend $=0.01$ ), and a smaller increase in steps during the intervention period (ambiguous impairment: -620 [95\% CI -174, -1,415]; MCI: $-1,653$ [95\% CI $-120,-3,187]$; $p$ for trend $<0.01$ ) (Table 3).

Association of Cognitive Function Scores with Change in Outcomes from 3 to 6 months

During the postintervention period, higher levels of impairment on the TICS were associated with a smaller increase in physical performance on the SPPB (ambiguous impairment: -0.54 [95\% CI $-1.27,0.19]$; MCI: -0.97 [95\% CI $-0.37,-1.58]$; $p$ for trend $<0.01$ ), and a smaller increase in self-reported physical function (ambiguous impairment: -3.3 [95\% CI -6.5, -0.04]; MCI: - 10.5 [95\%
CI $-18.7,-2.3]$; $p$ for trend $=0.04$ ). There was no association between cognitive impairment and change in steps between 3 and 6 months.

\section{Discussion/Conclusion}

Our study showed that participants' scores on a screening test for cognitive function were associated with both adherence and performance in a pedometer-based exercise intervention designed for patients with ESKD. Patients in this study showed a similar degree of cognitive impairment to that reported in other studies of patients treated with dialysis $[9,10]$. There was a significant correlation between baseline cognitive function and baseline physical performance. Participants with lower scores on cognitive function screening had lower rates of adherence than those with higher levels of function. Although patients in the intervention did increase the number of steps they walked during the 3 months of the intervention, those with lower scores on cognitive function screening increased their step counts little during this period than those without cognitive impairment. Patients with lower scores on cognitive function screening also demonstrated smaller increases in physical performance and reported less improvement in physical function during the intervention and the 3 -month postintervention follow-up period.

It is important to note that cognitive impairment is highly correlated with sarcopenia and other factors that are also associated with impairments in physical performance which may in turn lead to reduced performance in an exercise intervention [43-45]. Although our study 
showed a correlation between baseline scores on cognitive function screening and physical performance, there was no correlation between cognitive function and baseline step count or self-reported physical function. In addition, our analysis showed a strong association between lower results on cognitive function screening and improvement in performance even after controlling for baseline physical activity and physical function along with other factors such as known cerebrovascular disease. It seems unlikely that the reduced performance in the intervention among patients with cognitive impairment was related entirely to lower baseline physical functioning.

Indeed, another study by Uemura et al. [22] of a 6-month, twice weekly exercise intervention in older adults with MCI showed that adherence to the intervention and level of cognitive impairment were both associated with change in physical performance during their intervention. It is plausible that participants in our study with greater cognitive impairment also derived less benefit with regard to improvement in physical performance and self-reported physical function. One reason why less benefit was achieved may be in part be due to reduced adherence to weekly calls. The adherence of $89 \%$ reported in the Uemura study was similar to the adherence we observed in our study, and both were higher than the mean $53 \%$ adherence reported during other studies of moderate or lower intensity exercise in older adults with MCI [25]. The higher adherence among those with cognitive impairment in our study may be because our study included some of the factors associated with improving physical activity in older adults with MCI, as outlined by the Resources and Activities for Life Long Independence (RALLI) guidelines [24]. Specifically, our intervention included providing a log, demonstrating activity monitoring, setting specific goals, and reviewing logs of activity frequently. Indeed, overall adherence of the total cohort was higher than that reported in other studies of walking in ESKD [34, 46].

Although our intervention incorporated several strategies recommended to facilitate adherence among those who may face barriers, those with lower scores on cognitive function screening still demonstrated lower levels of adherence to the intervention. This lower adherence may have been related to increased difficulty with finding ways to incorporate walking into their schedule, or difficulty remembering scheduled times for counseling and feedback sessions. One longitudinal study of cognitive decline among patients with ESKD suggested that due to the burden of cerebrovascular disease in this population, execu- tive function may decline at faster rates than other cognitive domains [7]. To help mitigate this, the RALLI guidelines suggest that in addition to the above-mentioned practices, strategies to specifically increase remembering to do exercise, how to maintain momentum, and how to enjoy exercise should be incorporated [24]. The Seattle Protocols are a collective of programs designed to decrease disability and delay disease progression using a standardized approach towards exercise interventions among older adults with dementia or MCI [29]. These protocols additionally focus on the importance of making exercise pleasant to perform and teaching both participants and caregivers' successful strategies for reaching and maintaining exercise goals [29]. Including such practices in future studies may help improve adherence and potentially improve performance among patients with ESKD and cognitive impairment.

The potential importance of caregivers in scaling up such interventions cannot be overestimated. Prior studies of patients with ESKD show that many patients rely on caregivers [47-50] for many of their daily activities, and our own analysis of the goals and barriers reported by participants in the intervention showed that lack of caregiver participation was a perceived issue [32]. However, surprisingly few studies have addressed the importance of caregivers to the success of exercise interventions in elderly populations. At least one study suggests that exercise training for dialysis patients may improve QoL for the caregivers of such patients [51]. To our knowledge, no study has elicited caregiver input on the burden of such interventions on the caregiver or how the caregiver could assist the patient to successfully participate. Such information would be valuable for designing future studies.

Strengths of our study include the ability to control for baseline physical function as a potential confounder and detailed assessment of adherence and performance during the intervention and postintervention follow-up. Another strength is overall diversity - both in terms of racial and ethnic groups as well as dialysis modality; many studies focus on only in-center hemodialysis [2], and the possibility of selection bias (with more cognitively intact patients selecting PD), cannot be discounted [52].

One limitation of the study is the category of ambiguous impairment within the TICS itself, as patients falling into this category would be better characterized with more rigorous neuropsychological testing. However, in our study, there was a clear trend toward worse adherence and performance with lower TICS category, and even patients in the "ambiguous" category did not do as well as those who were clearly unimpaired. Future studies should 
consider more definitive follow-up testing for patients identified by TICS to be in the "ambiguous" category or consider alternate cognitive function testing that includes specific assessments of executive function. Another potential limitation of the study is that the TICS was administered during a dialysis session, and therefore there may have been outside distractions. However, prior studies have demonstrated that testing during the first hour of dialysis is equivalent to testing prior to dialysis and likely more convenient for patients in terms of scheduling [53]. Other limitations include that despite including patients with prior stroke and accounting for them in our analysis, we are not fully able to control for severity of existing vascular disease. Because of the nature of the intervention, the most cognitively impaired patients (those who would have been deemed unsuitable by dialysis staff or were unable to provide informed consent) were not enrolled. Indeed, no patient scored below a 21 on the TICS (moderate to severe cognitive impairment). We also excluded patients who were wheelchair-bound, since this was a pedometer-based intervention. This study also did not examine household income or other variables of socioeconomic status which may have explained some portion of the differences in adherence or performance in the intervention. Finally, study participants were selected from dialysis facilities in Northern California, which may limit generalizability of physical activity to the broader US dialysis population.

Patients with lower scores on cognitive function screening had worse adherence to a 3-month pedometerbased intervention, missing more weekly phone calls and having a lower likelihood of meeting the overall step goals. Patients with lower scores on cognitive function screening also derived less benefit from the intervention, experiencing smaller increases in physical performance and self-reported function at the end of the intervention and at a 3-month postintervention follow-up (6 months from the baseline) than those without cognitive impairment. Future exercise interventions should incorporate specific measures to mitigate potential cognitive impairment, which is highly prevalent in the ESKD population, including taking care to promote enjoyment of exercise and to involve caregivers.

\section{Statement of Ethics}

The PED study adheres to the Declaration of Helsinki, was approved by the UCSF Committee on Human Research, and was registered at ClinicalTrials.gov (NCT02623348).

\section{Conflict of Interest Statement}

None declared. The results presented in this paper have not been published previously in whole or in part.

\section{Funding Sources}

Dr. Sheshadri's effort was supported by an American Kidney Fund Clinical Scientist in Nephrology Fellowship and a Ruth L. Kirschstein National Research Service Award (NRSA) Individual Postdoctoral Fellowship (F32 DK111154-02). ClinicalTrials.gov ID NCT02623348. This publication was also supported by the UCSF Academic Senate Committee on Research. Dr. Kittiskulnam received support from an International Society of Nephrology fellowship. Dr. Johansen's effort was supported by a Midcareer Investigator Award in Patient Oriented Research (K24-DK085153). Dr. Delgado's work is supported by the Department of Veterans Affairs, Clinical Science Research and Development Program under Career Development Award 1IK2CX000527-01A2. Her contribution is the result of work supported with the resources and the use of facilities at the San Francisco VA Medical Center. Dr. Sudore was funded in part by the National Institute on Aging, National Institutes of Health (K24AG054415). Dr. Lai's effort was funded by NIH R01AG059183. None of the funders had a role in study design, data collection, analysis, reporting, or the decision to submit for publication.

\section{Author Contributions}

Research idea and study design: A.S., P.K., and K.J.; data acquisition: A.S. and P.K.; statistical analysis and interpretation: A.S.; manuscript drafting and revision: A.S., P.K., C.D., R.S., J.L., and K.J.: supervision and mentorship: J.L., R.S., and K.J. All authors contributed to the important intellectual content during manuscript drafting or revision and accepts accountability for the overall work by ensuring that questions pertaining to the accuracy or integrity of any portion of the work are appropriately investigated and resolved.

\section{References}

High KP, Brennan-Ing M, Clifford DB, Cohen MH, Currier J, Deeks SG, et al. HIV and aging: state of knowledge and areas of critical need for research. A report to the NIH office of AIDS research by the HIV and Aging Working Group. J Acquir Immune Defic Syndr. 2012;60(Suppl 1):S1-18.

2 Kittiskulnam P, Sheshadri A, Johansen KL. Consequences of CKD on functioning. Semin Nephrol. 2016;36(4):305-18.

3 Bugnicourt JM, Godefroy O, Chillon JM, Choukroun G, Massy ZA. Cognitive disorders and dementia in CKD: the neglected kidney-brain axis. J Am Soc Nephrol. 2013;24(3): 353-63.

4 da Matta SM, Moreira JM, Kummer AM, Barbosa IG, Teixeira AL, Silva ACS. Cognitive alterations in chronic kidney disease: an update. J Bras Nefrol. 2014;36(2):241-5. 
5 Weiner DE, Tabatabai S, Tighiouart H, Elsayed E, Bansal N, Griffith J, et al. Cardiovascular outcomes and all-cause mortality: exploring the interaction between CKD and cardiovascular disease. Am J Kidney Dis. 2006; 48(3):392-401.

6 Kurella M, Mapes DL, Port FK, Chertow GM. Correlates and outcomes of dementia among dialysis patients: the Dialysis Outcomes and Practice Patterns Study. Nephrol Dial Transplant. 2006;21(9):2543-8.

7 Drew DA, Weiner DE, Tighiouart H, Duncan S, Gupta A, Scott T, et al. Cognitive decline and its risk factors in prevalent hemodialysis patients. Am J Kidney Dis. 2017;69(6):780-7.

8 Shen Z, Ruan Q, Yu Z, Sun Z. Chronic kidney disease-related physical frailty and cognitive impairment: a systemic review. Geriatr Gerontol Int. 2017;17(4):529-44.

9 Kurella M, Chertow GM, Luan J, Yaffe K. Cognitive impairment in chronic kidney disease. J Am Geriatr Soc. 2004;52(11):1863-9.

10 Sehgal AR, Grey SF, DeOreo PB, Whitehouse PJ. Prevalence, recognition, and implications of mental impairment among hemodialysis patients. Am J Kidney Dis. 1997;30(1):41-9.

11 Madan P, Kalra OP, Agarwal S, Tandon OP. Cognitive impairment in chronic kidney disease. Nephrol Dial Transplant. 2007;22(2): $440-4$.

12 Griva K, Stygall J, Hankins M, Davenport A, Harrison M, Newman SP. Cognitive impairment and 7-year mortality in dialysis patients. Am J Kidney Dis. 2010;56(4):693-703.

13 Drew DA, Weiner DE, Tighiouart $\mathrm{H}$, Scott T, Lou K, Kantor A, et al. Cognitive function and all-cause mortality in maintenance hemodialysis patients. Am J Kidney Dis. 2015;65(2): 303-11.

14 Angermann S, Schier J, Baumann M, Steubl D, Hauser C, Lorenz G, et al. Cognitive impairment is associated with mortality in hemodialysis patients. J Alzheimers Dis. 2018; 66(4):1529-37.

15 Abbott RD, White LR, Ross GW, Masaki KH, Curb JD, Petrovitch H. Walking and dementia in physically capable elderly men. JAMA. 2004;292(12):1447-53.

16 Wueve J, Kang JH, Manson JE, Breteler MMB, Ware JH, Grodstein F. Physical activity, including walking, and cognitive function in older women. JAMA. 2004;292(12):1454-61.

17 Tudor-Locke C, Washington TL, Hart TL. Expected values for steps/day in special populations. Prev Med. 2009;49(1):3-11.

18 Johansen KL, Chertow GM, Kutner NG, Dalrymple LS, Grimes BA, Kaysen GA. Low level of self-reported physical activity in ambulatory patients new to dialysis. Kidney Int. 2010; 78(11):1164-70.

19 Johansen KL, Chertow GM, Ng AV, Mulligan K, Carey S, Schoenfeld PY, et al. Physical activity levels in patients on hemodialysis and healthy sedentary controls. Kidney Int. 2000; 57(6):2564-70.

20 Sheshadri A, Kittiskulnam P, Lazar AA, Johansen KL. A walking intervention to in- crease weekly steps in dialysis patients: a pilot randomized controlled trial. Am J Kidney Dis. 2020;75(4):488-96.

21 Lam FM, Huang MZ, Liao LR, Chung RC, Kwok TC, Pang MY. Physical exercise improves strength, balance, mobility, and endurance in people with cognitive impairment and dementia: a systematic review. J Physiother. 2018;64(1):4-15.

22 Uemura K, Shimada H, Makizako H, Doi T, Yoshida D, Tsutsumimoto K, et al. Cognitive function affects trainability for physical performance in exercise intervention among older adults with mild cognitive impairment. Clin Interv Aging. 2013;8:97-102.

23 Lofgren N, Conradsson D, Joseph C, Leavy B, Hagstromer M, Franzen E. Factors associated with responsiveness to gait and balance training in people with Parkinson disease. J Neurol Phys Ther. 2019;43(1):42-9.

24 Logsdon RG, McCurry SM, Pike KC, Teri L. Making physical activity accessible to older adults with memory loss: a feasibility study. Gerontologist. 2009;49(Suppl 1):S94-9.

25 Tak EC, van Uffelen JG, Paw MJ, van Mechelen W, Hopman-Rock M. Adherence to exercise programs and determinants of maintenance in older adults with mild cognitive impairment. J Aging Phys Act. 2012;20(1): $32-46$.

26 Sheshadri A, Johansen KL. Prehabilitation for the frail patient approaching ESRD. Semin Nephrol. 2017;37(2):159-72.

27 Johansen KL. Exercise in the end-stage renal disease population. J Am Soc Nephrol. 2007; 18(6): 1845-54

28 Johansen KL, Painter PL, Sakkas GK, Gordon P, Doyle J, Shubert T. Effects of resistance exercise training and nandrolone decanoate on body composition and muscle function among patients who receive hemodialysis: a randomized, controlled trial. J Am Soc Nephrol. 2006;17(8):2307-14.

29 Teri L, Logsdon RG, McCurry SM. Exercise interventions for dementia and cognitive impairment: the seattle protocols. J Nutr Health Aging. 2008;12(6):391-4.

30 Rimmer JH, Chen MD, McCubbin JA, Drum C, Peterson J. Exercise intervention research on persons with disabilities: what we know and where we need to go. Am J Phys Med Rehabil. 2010;89(3):249-63.

31 Delgado C, Johansen KL. Barriers to exercise participation among dialysis patients. Nephrol Dial Transplant. 2012;27(3):1152-7.

32 Sheshadri A, Kittiskulnam P, Delgado C, Sudore R, Lai JC, Johansen KL. Association of motivations and barriers with participation and performance in a pedometer-based intervention. Nephrol Dial Transplant. 2020; 35(8):1405-11.

33 Tudor-Locke C Jr, Bassett DR. How many steps/day are enough? preliminary pedometer indices for public health. Sports Med. 2004;34(1):1-8.

34 Nowicki M, Murlikiewicz K, Jagodzińska M. Pedometers as a means to increase sponta- neous physical activity in chronic hemodialysis patients. J Nephrol. 2010;23(3):297305.

35 Bravata DM, Smith-Spangler C, Sundaram V, Gienger AL, Lin N, Lewis R, et al. Using pedometers to increase physical activity and health: a systematic review. JAMA. 2007; 298(19):2296-304.

36 Hergenroeder AL, Barone Gibbs B, Kotlarczyk MP, Perera S, Kowalsky RJ, Brach JS. Accuracy and acceptability of commercial-grade physical activity monitors in older adults. J Aging Phys Act. 2019;27(2):222-9.

37 Brandt J, Specter M, Folstein M. The telephone interview for cognitive status. Neuropsychiatry Neuropsychol Behav Neurol. 1988;1:111-7.

38 Fong TG, Fearing MA, Jones RN, Shi P, Marcantonio ER, Rudolph JL, et al. Telephone interview for cognitive status: creating a crosswalk with the mini-mental state examination. Alzheimers Dement. 2009;5(6):492-7.

39 Ferrucci L, Del Lungo I, Guralnik JM, Bandinelli S, Benvenuti E, Salani B, et al. Is the telephone interview for cognitive status a valid alternative in persons who cannot be evaluated by the mini mental state examination? Aging. 1998;10(4):332-8.

40 Brandt J, Folstein MF. Telephone interview for cognitive status professional manual. Florida: Psychological Assessment Resources; 1987.

41 Guralnik JM, Simonsick EM, Ferrucci L, Glynn RJ, Berkman LF, Blazer DG, et al. A short physical performance battery assessing lower extremity function: association with self-reported disability and prediction of mortality and nursing home admission. J Gerontol. 1994;49(2):M85-94.

42 Hays RD, Kallich JD, Mapes DL, Coons SJ, Carter WB. Development of the kidney disease quality of life (KDQOL) instrument. Qual Life Res. 1994;3(5):329-38.

43 Kim JK, Choi SR, Choi MJ, Kim SG, Lee YK, Noh JW, et al. Prevalence of and factors associated with sarcopenia in elderly patients with end-stage renal disease. Clin Nutr. 2014; 33(1):64-8.

44 Kim E, Sok SR, Won CW. Factors affecting frailty among community-dwelling older adults: a multi-group path analysis according to nutritional status. Int J Nurs Stud. 2021; 115:103850.

45 Li F, Harmer P. Prevalence of falls, physical performance, and dual-task cost while walking in older adults at high risk of falling with and without cognitive impairment. Clin Interv Aging. 2020;15:945-52.

46 Manfredini F, Mallamaci F, D'Arrigo G, Baggetta R, Bolignano D, Torino C, et al. Exercise in patients on dialysis: a multicenter, randomized clinical trial. J Am Soc Nephrol. 2017;28(4):1259-68.

47 Urquhart-Secord R, Craig JC, Hemmelgarn B, Tam-Tham H, Manns B, Howell M, et al. Patient and caregiver priorities for outcomes in hemodialysis: an International Nominal 
Group Technique Study. Am J Kidney Dis. 2016;68(3):444-54.

48 Hoang VL, Green T, Bonner A. Informal caregivers' experiences of caring for people recieving dialysis: a mixed-methods systematic review. J Ren Care. 2018;44(2):88-95.

49 Cook WL, Jassal SV. Functional dependencies among the elderly on hemodialysis. Kidney Int. 2008;73(11):1289-95.
50 Farzi S, Farzi S, Moladoost A, Ehsani M, Shahriari M, Moieni M. Caring burden and quality of life of family caregivers in patients undergoing hemodialysis: a Descriptive-Analytic Study. Int J Community Based Nurs Midwifery. 2019;7(2):88-96.

51 Garcia RSA, Pinheiro BV, Lucinda LMF, Pimentel AL, Junior JMP, Paula RB, et al. Association between exercise training in haemodialysis patients and burden of their family caregivers: a cross-sectional study. Nephrology. 2020;25(4):332-8.
52 Radic J, Ljutić D, Radic M, Kovacic V, Sain M, Dodig Curkovic K. The possible impact of dialysis modality on cognitive function in chronic dialysis patients. Neth J Med. 2010; 68(4):153-7.

53 Drew DA, Tighiouart H, Scott TM, Lou KV Shaffi K, Weiner DE, et al. Cognitive performance before and during hemodialysis: a randomized cross-over trial. Nephron Clin Pract. 2013;124(3-4):151-8. 